\title{
Imaging Soft and Hard Dielectric Breakdown in Resistive Switching
}

B. C. Regan ${ }^{1}$, Jared Lodico ${ }^{1}$, Ho Leung Chan ${ }^{2}$, Matthew Mecklenburg ${ }^{3}$ and William Hubbard ${ }^{4}$

${ }^{1}$ Department of Physics and Astronomy, University of California, Los Angeles, California 90095, United States, United States, ${ }^{2}$ University of California, Los Angeles, Los Angeles, California, United States, ${ }^{3}$ Core Center of Excellence in Nano Imaging (CNI), University of Southern California, Los Angeles, California 90089, United States, United States, ${ }^{4}$ NanoElectronic Imaging, Inc., Los Angeles, California, United States

Dielectric breakdown (DB) controls the function and failure of billions of devices inside a modern computer [1]. In transistors and capacitors, DB represents a failure mechanism. In resistive random access memory (RRAM), DB is exploited as a functional mechanism: the RRAM sub-category valence change memory (VCM) specifically uses controlled DB in oxide films to store digital ' 1 's and '0's.

Despite DB's overwhelming importance in modern computing hardware, previously it has not been possible to visualize the microscopic physics of DB with good contrast in an oxide film. Under ideal imaging conditions, standard microscopy techniques can localize individual atoms, but they cannot distinguish an insulator from a conductor. And DB has much in common with doping a semiconductor: a tiny concentration change produces a conductivity change that is millions of times larger.

Here we present the first images showing contrast based on the big, directly relevant effect — the huge conductivity change - as opposed to the tiny, indirectly-relevant one. We image DB in live, cycling VCM devices using scanning transmission electron microscope (STEM) electron beam-induced current (EBIC) imaging. The devices are fabricated with a slant-vertical architecture [2] that preserves device function while allowing STEM imaging access, allowing devices to switch numerous times while imaging. Electronic changes during switching appear in the STEM EBIC images through both the "standard," electric field-sensitive EBIC mode and secondary electron emission EBIC (SEEBIC) [3]. The latter is new and visualizes conductivity directly $[3,4]$, with high spatial resolution and good contrast, and thereby sheds new light on the structures underlying DB.

Our results imply a model of DB in which every aspect - the soft filament, the hard filament, the charge sensitive conduction mechanism, and the large electric fields driving the DB process - is clearly revealed by a distinct and separable signal in the STEM EBIC images. These results give an unprecedented look at the electronic structure of a bulk insulator under stress. 


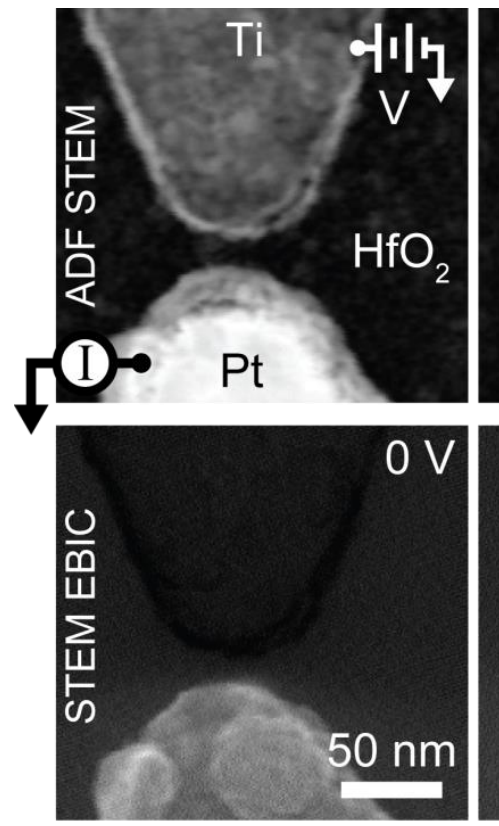

pristine device

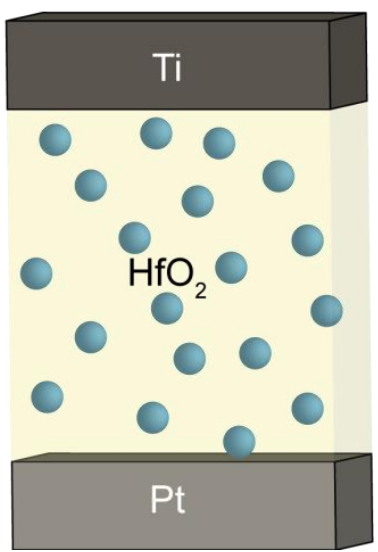

Figure 1. Figure 1 (Upper row) ADF STEM and (middle row) STEM EBIC images show a slant-vertical $\mathrm{Pt} / \mathrm{HfO} 2 / \mathrm{Ti}$ device biased through the pre-switching "forming" stage. With the Pt electrode grounded the Ti is biased at $0 \mathrm{~V}, 5 \mathrm{~V}, 10 \mathrm{~V}$, and then returned to $0 \mathrm{~V}$. STEM EBIC is measured on the Pt electrode. While biasing no changes are seen in the ADF STEM, but distinct features appear in the EBIC. As diagrammed in the lower row, $5 \mathrm{~V}$ bias produces a conducting regions of soft dielectric break down (the "soft" filament) that is detected in STEM EBIC (via SEEBIC) as a diffuse bright region growing from the Pt. With increasing bias the soft filament grows towards the Ti electrode. The large electric field generated by the increasing voltage and decreasing filament-Ti gap produces a very strong standard EBIC signal (bright spot). When voltage is returned to zero (rightmost images) the device resembles the pristine state in STEM EBIC, indicating these electronic changes are volatile.

\section{References}

[1] F Palumbo et al, Advanced Functional Materials 30 (2019) 1900657.

[2] WA Hubbard et al, Nano Letters 15 (2015) p. 3983-3987.

[3] WA Hubbard et al., Physical Review Applied 10 (2018), p. 044066.

[4] WA Hubbard et al., Applied Physics Letters 115 (2019), p. 133502.

[5] This work was supported by the Semiconductor Research Corporation, by National Science Foundation (NSF) award DMR-2004897, and by NSF STC award DMR-1548924 